\title{
Tentative Analysis on Application of Combination of Photogrammetry and GIS in Engineering Measurement
}

\author{
Hongxia Zhu \\ Chongqing Vocational Institute of Engineering, Chongqing 400037, China
}

Keywords: Photogrammetry, GIS, Engineering measurement, Specific application.

\begin{abstract}
The new field of engineering measurement can be carried out by combining GIS and photogrammetry. The combination of the two can sufficiently allocate existing engineering resources and provides necessary references for follow-up analyses. Besides, the mutual combination of GIS and photogrammetry offers a necessary new means of comprehensive analysis to gather, analyze and handle information and to provide decision-making reference. In this way, it can improve the comprehensive practical effect obtained through engineering measurement based on the lasting benefits and also promote the survey progress in the persistent state. Therefore, this paper analyzes the admissible GIS and frequently-used photogrammetry during engineering measurement and explores the specific application of their combination based on practical situations.
\end{abstract}

\section{Introduction}

In recent years, an increasing number of various projects have been put into construction; to enhance the overall project quality, it is required to properly control the daily engineering measurement. As a necessary link, engineering measurement relates to the construction progress in the overall scope and is a necessary construction reference. Concretely speaking, information available through engineering measurement includes drawings painted and all sorts of data obtained through measurement. It is required to analyze the most proper measurement process by combining the selected practical engineering situation so as to maximally lower the follow-up influence caused by a measurement deviation ${ }^{[1]}$. Only successful measurement can guarantee high-quality follow-up construction. In addition, with the trend of digitalization, GIS is increasingly used in engineering measurement and the this type of new technologies can be integrated into photogrammetry, so the real level of networked measurement can be fundamentally enhanced. The engineering measurement under the guidance of GIS obeys the development trend of digitalization, so the new photogrammetric technology can be accepted and generalized.

\section{Fundamental principle of photogrammetry and GIS}

Limited by multiple technologies, photogrammetry can only be used to identify and draw topographic maps for quite a long time. In such a condition, photogrammetry fails to be closely related to the novel microcomputer field. After development for a long time, the photogrammetry's morphological simulation measurement and traditional analysis measurement were discarded and a new connotation of digitalization was added. Besides, photogrammetry uses its own digitalization and is equipped with the software and hardware under the control of a microcomputer. Furthermore, photogrammetry can automatically generate diversified visual products and also expands its measurement performance to orthoimage, clear-structured surface model, virtual perspective drawing and other diagrams. The photogrammetry in diversified forms can be used in more projects and industries so the new measurement has an excellent prospect ${ }^{[2]}$.

Photogrammetric technology.

Over recent years, the application of normal state of microcomputer system has expanded the coverage and the new technologies of all subjects consist of the visual system with imagine processing, engineering mode identification, intelligent manual simulation and microcomputer control. With the development for a long time, photogrammetry exceeds its originally planned 
narrow scope and becomes more closely related to the microcomputer. Besides, in the condition of technological progress, the tedious and monotonous common manual control is abandoned in photogrammetry while the automaticity under the all-around structure is added in its measurement steps. Compared with the analytical measurement and simulation photogrammetry, the digitalized photogrammetry has the following differences: the initial information acquired through measurement is set as an image instead of a clear photo. During the measurement process, an additional large-scale light machine is not necessary because simulation and photography can be achieved only by the microcomputer.

Digital images can be divided into the space images and aerial images accordingly. Products obtained through photogrammetry include the surface model, describable surface features, orthoimage and topographic maps provided. In addition, the attached products generated by photography can also be expanded to the remaining intermediate products. The compound measurement products can be divided into the surface model, specific topographic map of digital grid and digital map etc. Compared with a single measurement product, the new product is constructed on basic space information and represents the external form of a grid. Therefore, the photogrammetry contains the vector information simultaneously, reduces the total cost and promotes the improvement of practical effects ${ }^{[3]}$.

\section{Measurement mode of GIS.}

The geographic information system (abbreviation: GIS) covers the environmental information and corresponding resource information, is constructed on and pays attention to the fundamental information technology, and integrates multiple disciplines so it shows higher comprehensiveness. An image of surface feature within the designated scope is available through analysis. With the assistance of photogrammetry and vectorized scanning, an analysis can precisely generate images. After such improvements, the digital information of a certain section at a certain time point can be judged out. The information of each section acquired by GIS shows the real-time property and higher precision so measurement shall be carried out according to the proposed indexes ${ }^{[4]}$. GIS also has the supporting functions of outputting patterns and controlling database etc. within the surveying and mapping scope so this technology becomes acceptable in many fields.

As for engineering measurement, GIS can process and obtain some information left by the database within a short time; such an imaging rate is very rapid and even exceeds the conventional measurement process. In this way, it can shorten the set processing progress and can help to easily acquire clear graphics and images so that the practical effect obtained through engineering design can also be enhanced accordingly. Meanwhile, GIS is additionally equipped with the auxiliary decision-making function and space information verifying or deploying function. As an auxiliary decision, GIS uses some information available for decision-making reference as follow-up reference. In the existing applications, this approach has been used to predict climate, speculate volcanic eruption, analyze and estimate the annual capacity of each kind of crop. Therefore, GIS can be preferred to obtain the clearest scale in agroforestry, transportation and national defense and other fields with diversified land resources.

\section{New technology through their combination}

\section{Technological combination under the new trend.}

With the macro technological progress, photogrammetry itself accelerates its original rate of progress. Digital photogrammetry follows the remote sensing technology under the new trend; the more matching algorithm can be adopted for judging the remote-sensing image, and more time phases are added for the combined remote-sensing image registration; the image itself has better resolution. For a certain measuring system selected, the way provided for remote-sensing processing has its necessary value. From the perspective of current development trend of remote sensing, remote-sensing image is added with inherent resolution and precision; the topographic map drawn is proposed to be 1:5000 in precision, and the image resolution can be set to be $1 \mathrm{~m}$ under the highest state. Under this state, with the help of the image, it should be possible to judge the qualitative state 
shown by a certain image; the original qualitative advantage is also added for calculation and measurement, and the updating of map data shortens the original overall cycle consumed. As for the supporting software and hardware, the photogrammetry and the currently new-style remote sensing combine closely into a complete unit ${ }^{[5]}$.

GIS integrates with digital photogrammetry, which is the trend in the current progress. With the integrated photogrammetry, GIS should be capable of obtaining necessary queryable information and getting the basic information that is related to space photography and used for updating of currently available data. Photogrammetry can be integrated in new technologies that are specific to GIS, showing the unique advantage of application integration itself. Onboard devices can record the accurate measuring position instantaneously, and the measured and calculated numerical values and information are entered into the photographic system; on this basis, the regional combination adjustment can be calculated. Photogrammetry can be used as the arithmetic operation representing the interior and exterior elements of orientation, and the stereoscopic impression of the model built is increased. From the perspective of progress trend, the ground control can be replaced with the new-style trigonometric survey.

GIS collects the superposable digital image, and it should be capable of obtaining various transcendental experiences from image dissection in the latter period. Collection and processing of engineering data rely on the microcomputer platform established; in this way, the single collection under the conventional path is changed, and the data stream established is added with the bidirectional characteristic ${ }^{[6]}$. Besides, DPS is also integrated in updating of various daily engineering information and adopts semi-automatic or higher-level automatic processing. GIS can obtain the inherently accurate height of a position or an object, for the purpose of gathering the accurate three-dimensional information. Thus, it can be seen that the remote-sensing image processing, the digital means selected for photogrammetry and the new-style system built by GIS are integrated into a complete unit that is impartible.

\section{Specific combination forms.}

Firstly, it is the novel remote sensing technology. The traditional remote sensing technology relies on aerial means and satellitic means etc. to remotely sense the emitted microwave and visible infrared light. As for a certain defined section, clear and precise images can be obtained through remote sensing aerial photography. With technical progress, the remote sensing technology gradually tends to have better intelligence and practical property and maximally enhanced the distinguishable probability. Accordingly, it is easier to identify the remote sensing spectrum, which clearly shows the internal diagnostic characteristics of spectrum. Compared with the frequently-used remote sensing means with a wide wave band, the new technology's unique advantage is outstanding. According to the current situation, the remote sensing measurement can not only be used to measure various projects in urban and rural construction but also be used to measure hydrology, land and traffic terrain. In this way, the queryable basis can be provided for set urban and rural measurement and planning ${ }^{[7]}$.

Secondly, it is the GIS combination. The two types of measurement under GIS integration can be divided into the dynamic and static measurement. As for the selected observation time interval, dual receivers have been provided to receive the engineering information to be measured. The measurement links under the combination state cover coordinate transmission, converted adjustment and converted coordinate. As for some projects equipped with the precise engineering control network, both GIS and photogrammetry can be adopted to enhance the measuring rate and the special measurement quality.

Then, it is GPS combination. The global position system follows the satellite navigation under the new trend, closely blends the three-dimensional and full-around new navigation, and integrates the air-sea-land diversified measurement modes with the help of navigation means. Compared with the common measurement, GPS adds the convenience during operation and enhances its inherent precision so it becomes more accurate and efficient. More importantly, GPS can get rid of bad intervisibility interference caused by climate and increase the flexibility, so it can be more widely used in the existing field ${ }^{[8]}$. Even though a project with poor intervisibility is selected, the attenuation 
precision is not significant. The flexible testing performance also expands the originally set operating radius and the imaging rate is accelerated during normal measurement with the help of GPS.

\section{Real engineering application}

\section{Gather original operating drawings.}

Digitalization is also added in the original operating drawing under the trend of combination of photogrammetry and GIS. Vectorized engineering scanning is mostly set in routine measurement because this scanning is highly convenient, efficient and extremely precise. Digitalized engineering processing is created for the selected map. The input method is set as a vectorized input method so that a topographic map can achieve its optimal accuracy.

During routine measurement, the imaging precision may still suffer various internal and external interferences including the tracking precision and equipment output precision, so measurement personnel should be able to know well the fundamental operating instructions and improve their consciousness of responsibility. In addition, normal measurement training shall be intensified and strictly implemented according to the proposed index. Guarantee the digitalized legend acquired through mapping is precise within the maximum range. Besides, the prepared map should be the most readable and distinguishable so that precise judgments can be made accordingly. Since some project itself is very comprehensive, it is required to provide measuring scales with different precisions to avoid repeated measurement ${ }^{[9]}$.

\section{Gather field data.}

It is required to select surface features and confirm the accurate codes when gathering all sorts of data about engineering field work. Besides, it is required to identify the necessary codes so that more potential deviations in the follow-up operations. During observation at the initial stage, the field workers shall strictly control observation in accordance with the given process and select the most proper observation site. Automatic mapping shall be able to describe the complete surface features and other representations. At first, portray a synoptic sketch to identify all sorts of surface features concerning in the project; then, describe mutual relations and the attributes of fragmentary points. As for the slope measurement, several topographic points on the lower side can be gathered. After a certain measuring zone is completed, go to the next designated measurement zone. If the condition allows, backup numerical values shall also be set.

\section{Depict and process interior work information.}

Compared with the field work processing, interior work information requires more measurement of adjustment. The most appropriate measuring mode should be selected at each phase of interior work. Besides, it is also required to prudently analyze the engineering result obtained through measurement and precisely handle the engineering advices available for reference. Information available through interior work processing should be saved in to floppy disk. Furthermore, clear drawing lines shall also be preserved by means of the plotting device. Ensure that lines drawn are mutually well-balanced and writings are neat and orderly. The externally exposed graphs shall show the optimal aesthetic perception and be kept tidy for the convenience of follow-up revisions. Repeated modification to an original graph is not allowed and it is required to ensure its timeliness.

\section{Conclusion}

Surveying and mapping supporting technologies are being perfected rapidly and outstanding long-acting progress has also been made on the new GIS technology acquired via improvement. The engineering information obtained from multisource shall be able to help to set the optimal engineering decision so as to guarantee successful analysis and processing. Both photogrammetry and the new-type engineering surveying adopted automatically set mapping steps so that multisource data under multiscale can be obtained. In today's surveying and mapping, it is also required to continuously enhance the real existing level of engineering measurement and repair the potential 
bugs and drawbacks. Photogrammetry under GIS control acquired the full-around measuring benefits and enhanced the surveying and mapping engineering level in the digitalized state.

\section{References}

[1] Yang Xingqiang. Study on application of geographic information system (GIS) technology in engineering measurement, Technology Innovation and Application, 2013 (10): 41.

[2] Mo Yougui. Study on application of modern surveying and mapping technology in engineering measurement, Guangdong Science and Technology, 2013 (12): 162+151.

[3] Lin Xiaoqing. Development of photogrammetry and remote sensing technology and their application prospects in engineering, Geomatics Technology and Equipment, 2011 (01): 37-38.

[4] Du Caihong. Application of UAV Photogrammetry in Ningxia Watercourse Engineering of the Yellow River, Shanxi Architecture, 2014 (32): 217-218.

[5] Li Chun. Discussion on the New Surveying and Mapping Technology Used in Engineering Survey, Guangdong Science \& Technology, 2012 (14): 292-293.

[6] Qin Changli. Expansion of engineering measurement in strategic leap of informationization surveying and mapping, Engineering Investigation, 2012 (12): 11-16.

[7] Huang Qifeng. Application of modern surveying and mapping technology in engineering measurement, Technology and Market. 2011 (05): 39-40.

[8] Chai Hongqi. Thinking and exploration on the development of engineering measurement technology, Industrial \& Science Tribune, 2011 (15): 93-94.

[9] Deng Deng. Development of GIS technology and digitalized surveying and mapping technology and their application in engineering measurement, Digital Technology and Application, 2014 (02): 110 . 